\title{
Optimal Conditions for Gas Cluster Ion Beams in Studying Inorganic Interface Species: Improved Chemical Information at a $\mathrm{ZnO}$ interface
}

\author{
Naoko Sano ${ }^{* 1}$, Anders J. Barlow ${ }^{1}$, Costas Tsakonas ${ }^{2}$, Wayne Cranton ${ }^{3}$, Peter J. Cumpson ${ }^{1}$ \\ ${ }^{1}$ National EPSRC XPS Users' Service (NEXUS), School of Mechanical and Systems Engineering, Newcastle University, \\ Newcastle upon Tyne, NE1 7RU, UK \\ ${ }^{2}$ College of Arts and Science, School of Science \& Technology, Nottingham Trent University, Burton Street, Nottingham, NG1 \\ 4BU, UK \\ ${ }^{3}$ Faculty of Arts, Computing, Engineering and Sciences, Sheffield Hallam University, Howard Street, Sheffield, S1 1WB, UK \\ E-mail address: naoko.sano@ncl.ac.uk
}

\begin{abstract}
We have observed for the first time a subtle chemical change in the oxidation state of $\mathrm{Zn}$ at the interface between a $\mathrm{ZnO}$ thin film and a glass substrate using gas cluster ion beam (GCIB) depth-profiling. A combination of monoatomic and gas clusters were used for etching, allowing the removal of the surface rapidly using the monoatomic ion beam yet still yield a less damaged surface by removing the damaged layer using the argon GCIB as a final step. With this combined method the depth-profile shows the transition of $\mathrm{Zn}$ from oxide to metal at the interface, which the monoatomic ion source if used alone would damage. This data indicates that the initial layers of the film did not undergo as much oxidation during the deposition compared to the rest of the film, therefore metallic zinc resulted at the interface. We also examined the $\mathrm{ZnO}$ thin film under a range of conditions of argon ion profiling from monoatomic to cluster ions to study the most efficient method for depth-profiling of multiple layers of inorganic materials. The monoatomic ion beam does not show the transition phenomena at the interface which was observed in cluster depth-profiling, probably due to atomic mixing effects. This suggests care be taken in selection of etch parameters when studying interfaces. We recommend the combination of monoatomic sputtering followed by GCIB etching as the most efficient method for looking at interfaces of inorganic thin layers from the point of view of time taken and minimising damage at interface.
\end{abstract}

KEYWORDS; Sputtering damage, Ar Gas Cluster ion beam etching, Monoatomic-GCIB mode, ZnO film, XPS

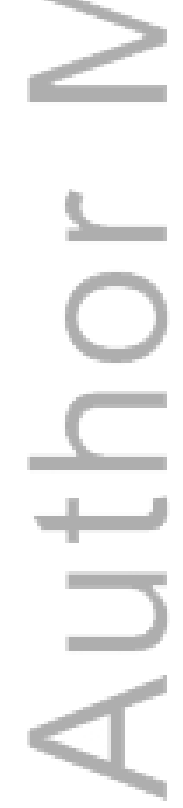

This is the author manuscript accepted for publication and has undergone full peer review but has not been through the copyediting, typesetting, pagination and proofreading process, which may lead to differences between this version and the Version of Record. Please cite this article as doi: 10.1002/sia.5949

This article is protected by copyright. All rights reserved. 


\section{INTRODUCTION}

Zinc Oxide $(\mathrm{ZnO})$ layers have attracted much attention as potential transparent semiconductor materials for applications in electronics, photonics, acoustics and sensing. On the electronic side, $\mathrm{ZnO}$ is competitive with other existing technologies. Nevertheless, $\mathrm{ZnO}$ still has some potential to develop in applications to transparent thin film transistors (TFTs) owing to its high optical transmittivity, high conductivity and relatively low production cost compared with GaN[1]. ZnO TFTs have improved device performance, mainly due to carrier mobility, while requiring only lower temperature or even room temperature processing in production[2]. Despite this promise, there are still a number of issues that are in need of further investigation. For instance, the following needs to improve for future devices before $\mathrm{ZnO}$ material can be transitioned to commercial use for these key applications:

- fabrication with $\mathrm{ZnO}$ needs to result in a highly smooth surface and amorphous structures,

- processing at low temperatures to facilitate the use of low cost needs to be demonstrated and

- shock resistant flexible substrates need to be developed.

To support these improvements, depth information is critically important to verify the nature of trial samples. Ion sputter depth-profiling has been widely used with X-ray photoelectron spectroscopy (XPS), Auger electron spectroscopy (AES) and secondary ion mass spectrometry (SIMS) for the characterisation of such thin film structures. Historically, monoatomic argon ion beam sources have been used to etch sample surfaces. However, the sputtering process can induce surface roughness and implant argon atoms, all of which contributes to degrading the resolution as a function of depth. In addition, the process also causes mixing of atoms within the samples by the ion beam. In recent years, argon Gas Cluster Ion Beam (GCIB) sources have become popular in XPS and SIMS instruments due to the minimal chemical damage that they typically introduce[3], especially in the analysis of polymer and organic materials[4]. GCIB sputtering may also be useful for inorganic materials such as semiconductors due to the lower roughness expected in cluster ion beam sputtering compared to that introduced by monatomic ion beams[5][6][7]. We have previously considered the total sputter yield of GCIB depth-profiling of organic/inorganic interfaces[8][9]. Here we examine in detail a model inorganic/inorganic interface.

In this work, our interest in $\mathrm{ZnO}$ thin films has been directed toward not only the chemical bond information from the transition regions of $\mathrm{ZnO} /$ glass structures but also the possibility of greater understanding of the ways in which GCIB sputtering may usefully be applied to this problem. We especially propose a conventional etching mode with an Ar monoatomic ion beam being followed with GCIB etching of the thin $\mathrm{ZnO}$ film. The proposed mode is a combination of selecting a 4,000 eV monoatomic ion beam for rapid etching and a 6,000 eV GCIB with approximately 1,000 atoms/cluster for removing damage at the surface. This mode allows us to remove most of the layer rapidly using the monoatomic ion beam, yet minimise the damage to the surface in the final step. If this mode is efficient for depth-profiling of inorganic thin films, it will dramatically save analysis time. Additionally, this mode will also provide more representative surface information due to the minimised surface damage. We have shown previously that GCIB treatment can induce damage in some inorganic materials[10], nevertheless in the majority of cases we should expect GCIB treatment to be much less damaging than monatomic sputtering.

As a part of this work, we have examined $\mathrm{ZnO}$ thin films under a range of conditions of argon ion profiling from monoatomic to cluster ions to study the most efficient method for depth profiling for multiple layers of 
these inorganic materials: monoatomic only $(4,000 \mathrm{eV})$, lower energy and larger clusters $(6,000 \mathrm{eV}$, approx.1000 atoms), and higher energy and smaller clusters (8,000 eV, approx. 300 atoms).

\section{Experimental}

\section{Materials ( $\mathrm{ZnO}$ films)}

Thin layers of $\mathrm{ZnO}$ were grown on microscope slides with no separate substrate heating. The substrate temperature attained on all depositions was no more than $80^{\circ} \mathrm{C}$. The sputtering system had a base pressure of $1.2 \times 10^{-7}$ Torr and the distance between the substrate and the target was around $100 \mathrm{~mm}$. A three inch $\mathrm{Zn}$ (99.99\%) metal target (Testbourne Ltd, Basingstoke, UK) was used as the source material. Two gas lines, $100 \%$ argon and $2 \%$ oxygen/argon, regulated by separate mass flow controllers provided the necessary sputter gas environment. The total sputter pressure was kept at 2 mTorr during the deposition. The $\mathrm{ZnO}$ films in this study were deposited using $300 \mathrm{~W}$ rf power and in a $0.7 \%$ oxygen in argon sputtering environment. The thickness of deposited films was measured with a stylus profilometer to be around $160 \mathrm{~nm}$. The film growth took 9 minutes 30 seconds.

\section{XPS analysis}

The XPS analyses were performed using a K-Alpha XPS instrument (Thermo Scientific, East Grinstead, UK) equipped with a monochromatic Al Ka X-ray source $(1486.6 \mathrm{eV})$. Pass energy was set at $40 \mathrm{eV}$ for high resolution spectra with $0.1 \mathrm{eV}$ step size and $200 \mathrm{eV}$ for survey spectra with $1.0 \mathrm{eV}$ step size. An X-ray spot size of $400 \mu \mathrm{m}$ diameter was selected to record both types of spectra. The K-Alpha is equipped with an argon GCIB gun (a Thermo MAGCIS ion gun model), giving access to a wide range of modes for etching conditions with different cluster sizes, including monoatomic, and beam energies from $1-10 \mathrm{keV}$. In this study, three modes were selected; argon monoatomic at 4,000 eV, approximately 300 atoms/cluster at 8,000 $\mathrm{eV}$ and approximately 1,000 atoms/cluster at 6,000 eV. Sputter yields of each mode are estimated as 5.0 Y/n, $0.4 \mathrm{Y} / \mathrm{n}$ and $0.002 \mathrm{Y} / \mathrm{n}$, respectively. For the estimation, the $\mathrm{ZnO}$ film sputter yield was regarded to be similar to silicon dioxide and Cumpson's sigmoidal sputter yield equation was used for the calculation[11]. For the etching, $1 \mathrm{~mm} \times 2 \mathrm{~mm}$ raster size was used in all measurements. The XPS spectra were processed using the CasaXPS software (Casa Software Ltd., Teignmouth, UK).

\section{Results and Discussion Ar monoatomic mode}

An Ar monoatomic ion beam at 4,000 eV was employed in our first experiment to observe the photoelectron spectra of the $\mathrm{ZnO}$ film as a part of the depth-profile. Figure 1 illustrates the three particular levels in this depth-profile with narrowscan spectra of the $\mathrm{Zn} 2 \mathrm{p}, \mathrm{O} 1 \mathrm{~s}$ and $\mathrm{Si} 2 \mathrm{p}$ energy ranges; (a) for the $\mathrm{ZnO}$ region of the film, (b) for the interface region between $\mathrm{ZnO}$ and the glass substrate, and (c) for the glass substrate. At the interface region, the Zn2p spectrum shows unusual doublet peaks as can be seen clearly in Figure 1 (b). These lower kinetic energy peaks are unlikely to be due to chemical shifts given the large $\sim 3 \mathrm{eV}$ energy difference. More likely is differential charging or extra-atomic relaxation[10] due to a mixing effect by the relatively high energy monoatomic argon ions. Alternatively, the interface may have been roughened due to the etching and the glass substrate; being an insulator, led to differential charging across the analysis area. To shed further light on this question, a $\mathrm{ZnO}$ film on a piece of silicon wafer was also examined under the same XPS instrumental conditions, however, this doublet peak was not observed at all. Consequently, the doublet phenomenon at the interface is confirmed to happen between the $\mathrm{ZnO}$ film and the glass substrate such that we may expect similar behaviour for other thin electro-conductive materials deposited on insulating or low conductivity materials. This phenomenon is a major impediment to understanding the nature of the $\mathrm{ZnO}$ film interface. 
To avoid misunderstanding of the nature of the $\mathrm{ZnO}$ film interface, an Ar GCIB was selected in the gas cluster mode with 300 atoms at a somewhat higher energy, specifically 8,000 eV. Generally speaking, this mode $(\sim 27 \mathrm{eV} / \mathrm{atom})$ is not gentle enough for material surfaces compared with lower energies-per-atom modes, such as 1000 atoms at 4,000 eV (4 eV/atom) which is popularly used for depth profile without destroying chemical bonds in organic materials[8][12]. Therefore, the clusters with 300 atoms at 8,000 eV mode may be expected to introduce some damage in organic materials and therefore may not be generally desirable. However, it is expected to be much more effective for depth-profiling the $\mathrm{ZnO}$ film due to the higher energy-per-atom of the cluster atoms. Besides, it is also anticipated to introduce less damage at the interfacial region and give better depth resolution than the monoatomic ion mode. Having said that, the analysis duration time is also important for most analysts, since the Ar GCIB gives much slower etching rates for inorganic materials than the monoatomic ion beam[3]. For these reasons, the following two modes were considered to be most effective in the depth-profiling of the $\mathrm{ZnO}$ film sample:

1) Ar gas cluster ion beam at 8,000 eV with 300 atoms/cluster (GCIB at $8 \mathrm{keV}, 300$ atoms mode), and

2) Ar monoatomic ion beam at $4,000 \mathrm{eV}$ and then gas cluster ion beam at $6,000 \mathrm{eV}$ with 1,000 atoms/cluster for 180 seconds (Monoatomic+GCIB mode).

This combination of the monoatomic and cluster beam succeeded in reducing the duration of the depthprofile with less damage to the surface. As described in the introduction section, the idea of the combination method for the $\mathrm{ZnO}$ films is to remove most of the layer rapidly using the monoatomic ion beam and these obtain a less damaged surface resulting from removing the damaged surface using the argon GCIB. The actual surface status following the combination mode is still to be confirmed, nevertheless it is expected that the damage layer is much less than the information depth of XPS with the advantage of shorter experimental time[4]. For instance, the combination mode took less than $1 / 25^{\text {th }}$ as much time as the GCIB at $8 \mathrm{keV}, 300$ atoms mode did in profiling these $\mathrm{ZnO}$ films without any doublet peaks observed as damage.

\section{GCIB at $8 \mathrm{keV,} 300$ atoms mode and Monoatomic+GCIB mode}

During the etching, no doublet peak like the spectra observed during monoatomic sputtering (shown in Figure 1 (b)) was observed, whether using either the GCIB at $8 \mathrm{keV}, 300$ atoms/cluster or the sequential monoatomic and GCIB mode. This confirms the observed shift in the spectra with the monoatomic mode is induced by sputtering, and it is considered that the action of the monoatomic ions causes the effect of differential charging at the interface by creating a 'rough' surface, rather than simply a result of the X-ray source.

Regarding efficiency of the analysis procedure from the point of running XPS instruments, it is considerably longer to sputter through the $\mathrm{ZnO}$ film using the GCIB with the 300 atoms/cluster at $8 \mathrm{keV}$ mode, although the results provided excellent data to confirm the presence of the metallic $\mathrm{Zn}$ layer. The etching duration time for reaching the interface was over 12 hours, and the total analysis duration was more than two days to collect the whole spectral dataset. The combination mode, Monoatomic+GCIB mode, reduced the sputter time dramatically but still retained the chemistry of the interface. As described above, the Zn LMM spectrum using this mode also shows the transition of $\mathrm{Zn}$ from oxide to metallic $\mathrm{Zn}$ at the interface in the same way as the GCIB depth-profile at $8 \mathrm{keV}, 300$ atoms/cluster did (see Figure 2). The etch duration was approximately 90 minutes to reach the interface and the total duration was less than six hours in the same conditions as above. Consequently, we recommend the combination mode, that is, a monoatomic ion beam at 4,000 eV followed by using GCIB with 1000 atoms/cluster at $6,000 \mathrm{eV}$ for 180 seconds as the efficient procedure for these inorganic layers.

The binding energies of zinc oxide and metallic zinc are subtlety different in the $\mathrm{Zn} 2 \mathrm{p}$ region, hence the LMM transition near the interface was of particular interest. Figure 2 illustrates Zn LMM spectra at the interface of the $\mathrm{ZnO}$ film using the two modes. Na KLL ( $\mathrm{Na}$ being one of the glass substrate) overlaps $\mathrm{Zn}$ 
LMM, therefore spectra of $\mathrm{Na}$ KLL, metallic $\mathrm{Zn} \mathrm{LMM}$ and zinc oxide $\mathrm{Zn} \mathrm{LMM}$ are also shown as references in Figure 2. The spectra at the interface region analysed using both modes show a metallic $\mathrm{Zn}$ peak at $991 \mathrm{eV}$. This result clearly indicates the $\mathrm{ZnO}$ film interface possesses a thin metallic $\mathrm{Zn}$ layer, probably forming a monolayer, as the initial layer on the glass substrate. This suggests that the initial layer of the film did not undergo as much oxidation during the deposition as the rest of the film.

The XPS technique using the Ar GCIB shows this result indicating a monoatomic layer of metallic $\mathrm{Zn}$ can be identified at the interface and this could not be identified using the monoatomic ion beam only. Indeed, we have observed for the first time a subtle chemical change in the oxidation state of $\mathrm{Zn}$ at the interface between the $\mathrm{ZnO}$ thin film and the glass substrate using both the GCIB mode and the combination mode. The ability to identify such a thin layer is important for the understanding and ultimate improvement of the functionality of $\mathrm{ZnO}$ films for use as transparent semiconducting materials. It is therefore clear that using GCIB is valuable for not only soft organic materials but also thin inorganic layers. Additionally, we expect it to be just as valuable for other metallic monolayers on insulating substrates to obtain precise information of the interface for atomic layer depositioned (ALD), for example. In ALD samples, not shown here, we have observed materials like gold thin layers on chromium and germanium to exhibit similar doublet peak phenomena at the interface when using the monoatomic ion beam for etching.

\section{Conclusions}

The nature of the interface of $\mathrm{ZnO}$ films was studied using XPS, moreover ion beam damage and its effects on XPS spectra was also studied. We have observed a subtle chemical change in the oxidation state of $\mathrm{Zn}$, likely due to an approximately monoatomic layer of metallic zinc at the interface between the $\mathrm{ZnO}$ thin film and the glass substrate using the GCIB depth-profiling modes. The monoatomic ion beam, if used alone, either damage or causes sample charging.

The authors have also examined the $\mathrm{ZnO}$ thin film under a range of conditions of argon ion profiling from monoatomic to cluster ions to study the most efficient method for depth-profiling of multiple layers of inorganic materials: monoatomic only $(4,000 \mathrm{eV})$, lower energy and larger clusters $(6,000 \mathrm{eV}$, approx. 1000 atoms/cluster), and higher energy and smaller clusters (8,000 eV, approx. 300 atoms/cluster). The results show that the monoatomic ion beam does not provide the transition phenomena at the interface observed in cluster depth-profiling, probably due to the atomic mixing effect. This indicates that one needs to be careful in selection of operating modes for depth-profiling when interfaces are the main focus of interest. In conclusion, we recommend the combination monoatomic and GCIB as the most efficient method to observe at interfaces of thin layers on substrates from the point of view of time spent and minimising any damage at interfaces.

\section{Acknowledgements}

X-ray photoelectron spectra were obtained at the National EPSRC XPS Users' Service (NEXUS) at Newcastle University, an EPSRC Mid-Range Facility. The authors gratefully acknowledge Dr Jose Portoles from NEXUS for helpful discussions and thank to Mr Mike Foster for his great support.

\section{REFERENCES}

[1] U. Ozgur, D. Hofstetter, H. Morkoç, Proceedings of the IEEE, 2010;7, 1225.

[2] E. Fortunato, P. Barquinha, R. Martins, Advanced Materials, 2012; 24,2945.

[3] S. Ninomiya, Y. Nakata, K. Ichiki, T. Seki, T. Aoki, J. Matsuo. Nucl. Instr.Meth. B. 2007; $256,493$.

[4] T. Miyayama, N. Sanada, S.R. Bryan, J.S. Hammond, M. Suzuki, Surf. Interface Anal. 2010; $42,1453$.

[5] J. D. P. Counsell, A. J. Roberts, W. Boxford, C. Moffitt, K. Takahashi, J. Surf. Anal. 2014; 20, 211. 
[6] R. Steinberger, J. Walter, T. Greunz, J. Duchoslav, M. Arndt, S. Molodtscov, D.C. Meyer, D. Stifter, Corrosion Science, 2015 in press, doi/10.1016/j.corsci.2015.06.019

[7] C. Deeks, P. Mack, Thermo Scientific Application Note 52606, accessed 4 September 2015.

<http://www.thermoscientific.com/content/dam/tfs/ATG/CAD/CAD\%20Documents/Application\%20\&\%20Technical\% 20Notes/Surface\%20Analysis/AN52476_E_1113M_H.pdf>

[8] A. J. Barlow, J. F. Portoles, and P.J. Cumpson, J. Appl. Phys. 2014; 116, 054908.

[9] P.J. Cumpson, J.F. Portoles, N. Sano, A.J. Barlow, , Surf. Interface Anal. 2013; 45, 1859.

[10] M.S. Raven, Surf. Int. Anal. 1979; 1, 20.

[11] P.J. Cumpson, J.F. Portoles, A.J. Barlow, N. Sano, J Appl. Phys. 2013;114, 124313.

[12] P.J. Cumpson, J.F. Portoles, N. Sano, J. Vac. Sci. Technol. A 2013; 31, 020605.

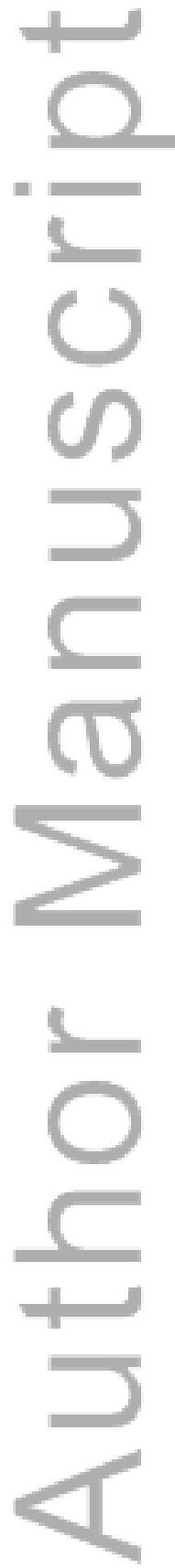



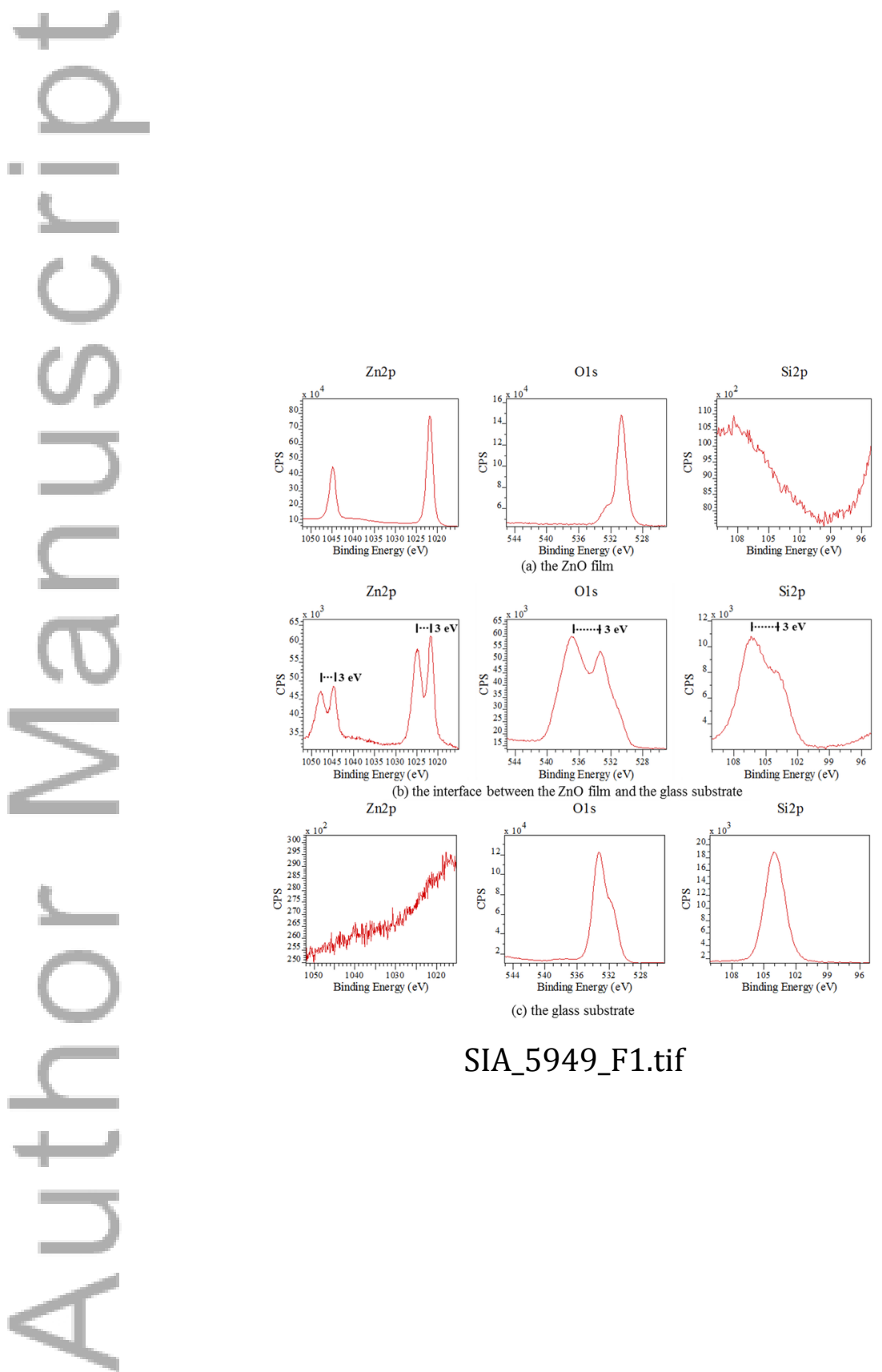

SIA_5949_F1.tif

This article is protected by copyright. All rights reserved. 


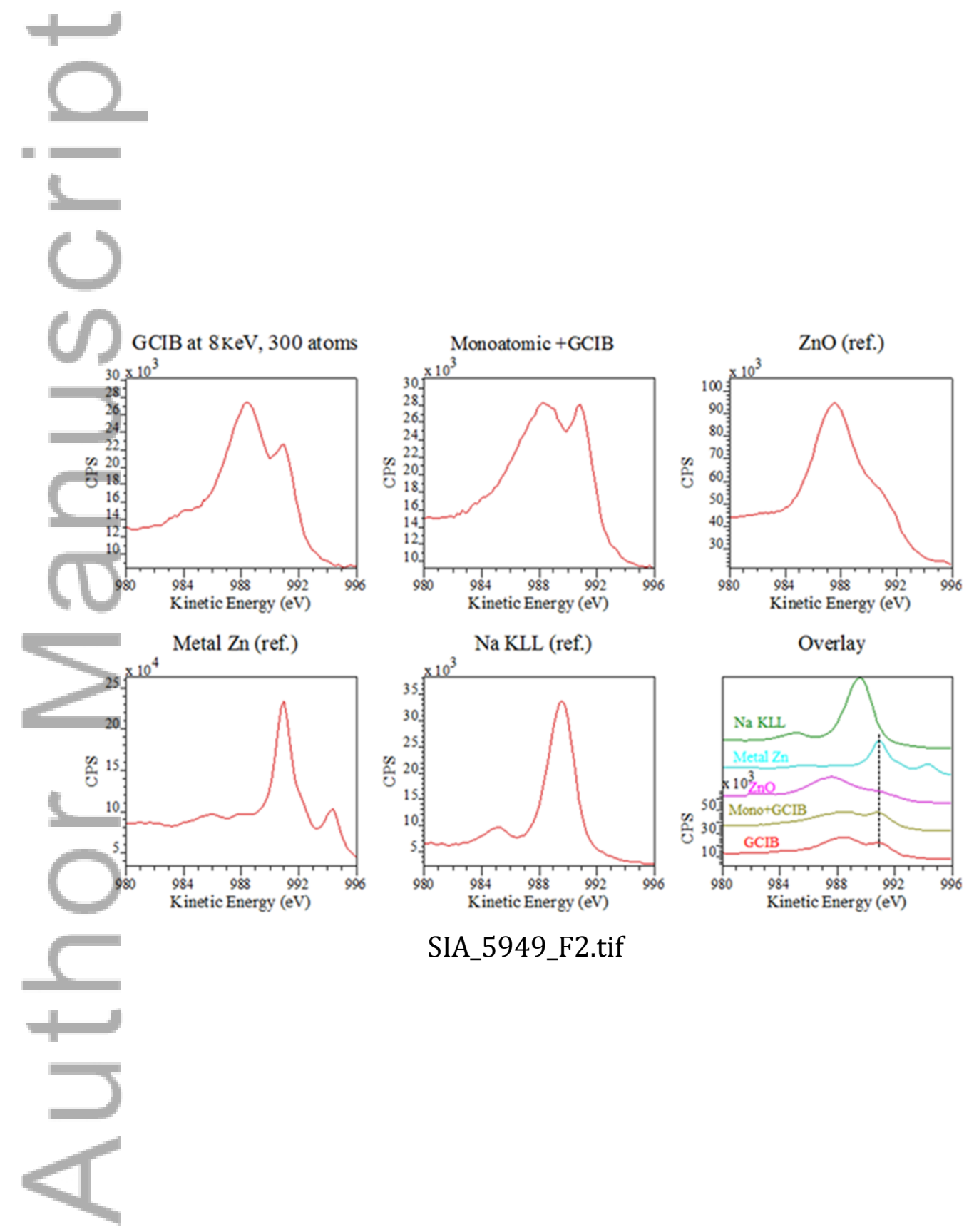

This article is protected by copyright. All rights reserved. 


\section{University Library}

\section{- M M N E R VA A gateway to Melbourne's research publications}

Minerva Access is the Institutional Repository of The University of Melbourne

Author/s:

Sano, N;Barlow, AJ;Tsakonas, C;Cranton, W;Cumpson, PJ

Title:

Optimal conditions for gas cluster ion beams in studying inorganic interface species: improved chemical information at a $\mathrm{ZnO}$ interface

Date:

2016-07-01

Citation:

Sano, N., Barlow, A. J., Tsakonas, C., Cranton, W. \& Cumpson, P. J. (2016). Optimal conditions for gas cluster ion beams in studying inorganic interface species: improved chemical information at a ZnO interface. SURFACE AND INTERFACE ANALYSIS, 48, (7), pp.575-579. WILEY. https://doi.org/10.1002/sia.5949.

Persistent Link:

http://hdl.handle.net/11343/290891 